Supporting Information

\title{
Self-assembly, binding and dynamic properties of heterodimeric porphyrin macrocycles
}

Pablo Ballester, ${ }^{*}{ }^{\dagger}$ Antoni Costa, ${ }^{\ddagger}$ Pere M. Deyà, ${ }^{\ddagger}$ Antonio Frontera, ${ }^{\ddagger}$ Rosa M. Gomila, ${ }^{\ddagger}$ Ana I. Oliva, ${ }^{\&}$ Jeremy K. M. Sanders ${ }^{\S}$ and Christopher A. Hunter ${ }^{*}$.

${ }^{\dagger}$ ICREA and Institute of Chemical Research of Catalonia (ICIQ), Avgda. Països Catalans $s / n, 43007-T a r r a g o n a$

${ }^{*}$ Departament de Química, Universitat de les Illes Balears, 07122-Palma de Mallorca, Spain

${ }^{\&}$ Institute of Chemical Research of Catalonia (ICIQ)

${ }^{\S}$ University Chemical Laboratories, Lensfield Road, Cambridge CB2 1EW, UK

${ }^{9}$ Centre for Chemical Biology, Krebs Institute for Biomolecular Science, Department of Chemistry, University of Sheffield, Sheffield S3 7HF, UK

E-mail: pballester@iciq.es

Contents

1. General Experimental

2. Figure S1. UV-vis spectra recorded during a titration of zinc bisporphyrins 4 a with bispyridine $\mathbf{3 a}$ in chloroform.

3. Figure S2. Selected regions of the ${ }^{1} \mathrm{H}$ NMR spectra recorded during a titration of zinc bisporphyrin $\mathbf{4 a}$ with bispyridine $\mathbf{3 a}$ in $\mathrm{CDCl}_{3}$ at $295 \mathrm{~K}$.

4. Figure S3. Expansion of a ROESY experiment performed on the self-assembled heterodimeric macrocycle 1aa.

S6

5. Figure S4. Portion of the aromatic region of the ${ }^{1} \mathrm{H}$ NMR spectra recorded during a titration of 1aa with diamide $\mathbf{8}$. 
6. Figure S5. Selected expansions of a ROESY experiment performed on the complex formed by macrocycle 1aa and diamide $\mathbf{8}$.

7. Figure S6. ${ }^{1} \mathrm{H}-\mathrm{NMR}$ spectrum of $\mathbf{3 a}$ in $\mathrm{CDCl}_{3} \quad$ S9

8. Figure S7. ${ }^{1} \mathrm{H}-\mathrm{NMR}$ spectrum of $\mathbf{4 a}$ in $\mathrm{CDCl}_{3} \quad \mathrm{~S} 10$

9. Figure S8. ${ }^{1} \mathrm{H}-\mathrm{NMR}$ spectrum of $\mathbf{1 a a}$ in $\mathrm{CDCl}_{3} \quad \mathrm{~S} 11$ 
General Experimental. All commercial reagents, unless otherwise noted, were reagent grade and used without further purification. Methylene chloride and triethylamine were distilled from $\mathrm{CaH}_{2}$ under nitrogen. Diethylamine and $N, N$-diisopropylethylamine were distilled from $\mathrm{KOH}$. Melting points are uncorrected. ${ }^{1} \mathrm{H}-\mathrm{NMR}$ spectra were recorded at 300 MHz. High resolution mass spectra were obtained using electron spray ionization (ESI MS). Deutero-chloroform and chloroform were deacidifed by passing through an alumina column.

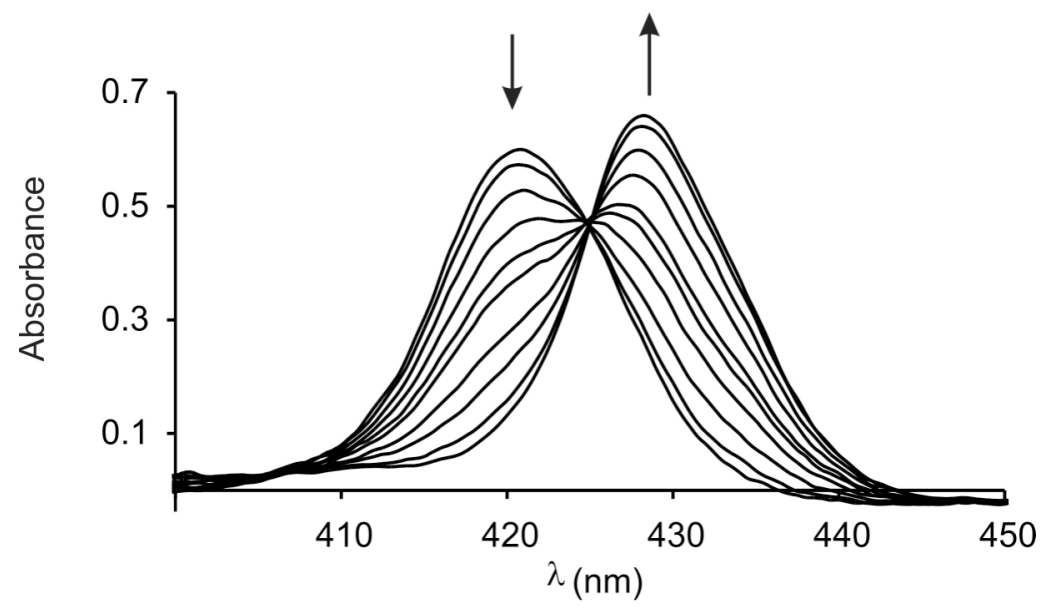

Figure S1. UV UV-vis spectra recorded during a titration of zinc bisporphyrin $\mathbf{4 a}$ with bispyridine 3a in chloroform (0 -16 equivalents of 3a) showing the existence of an isobestic point and the shift of the Soret band from 420 to $430 \mathrm{~nm}$. 

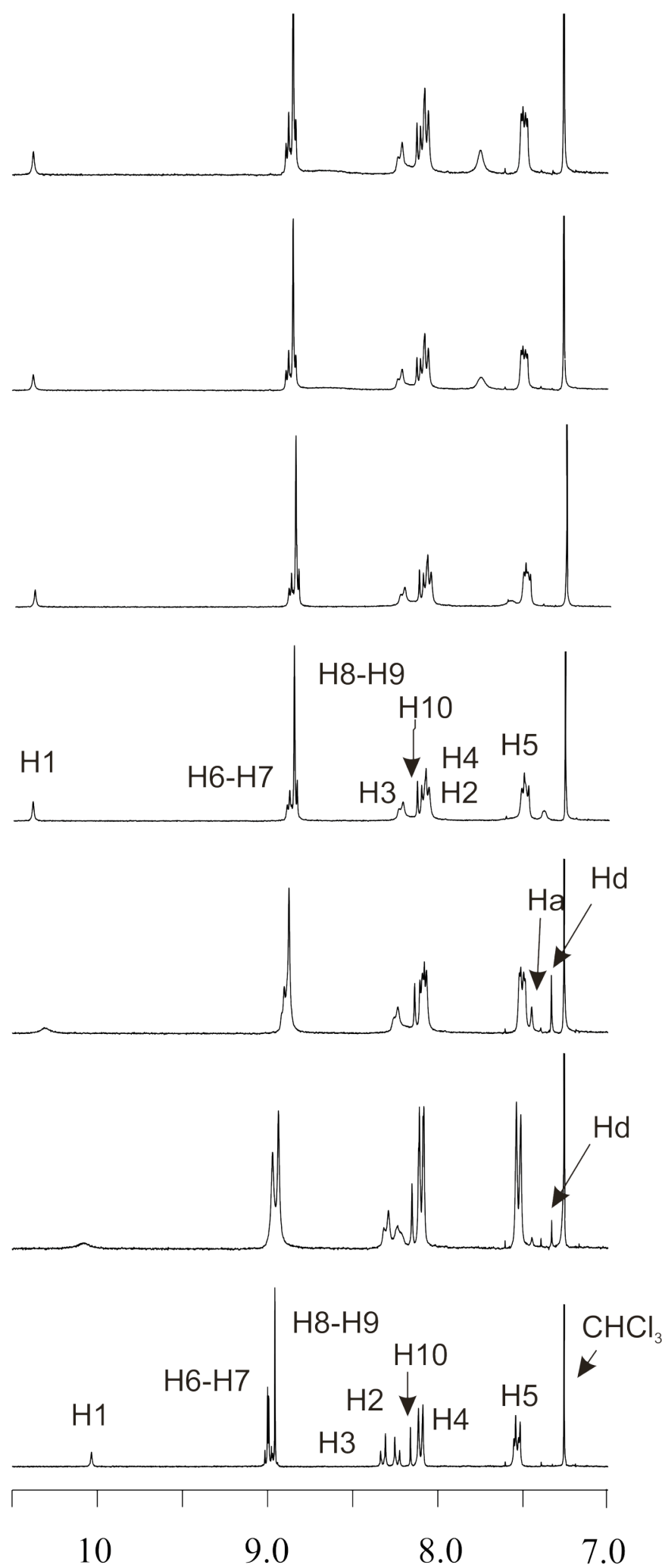
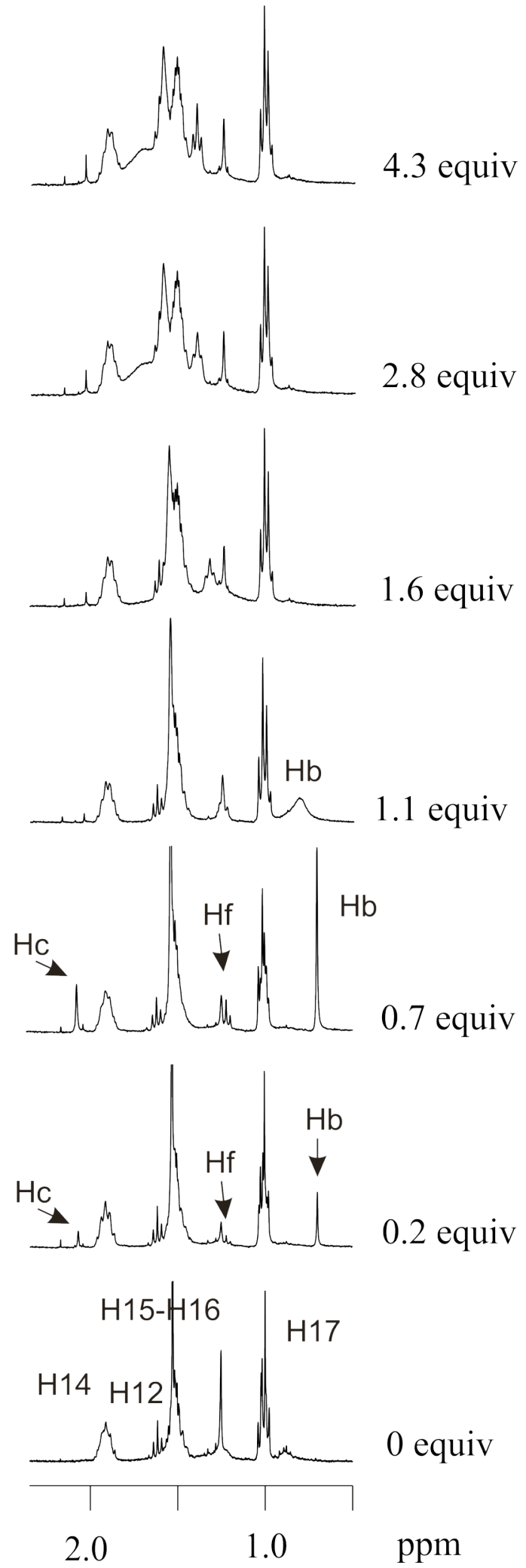

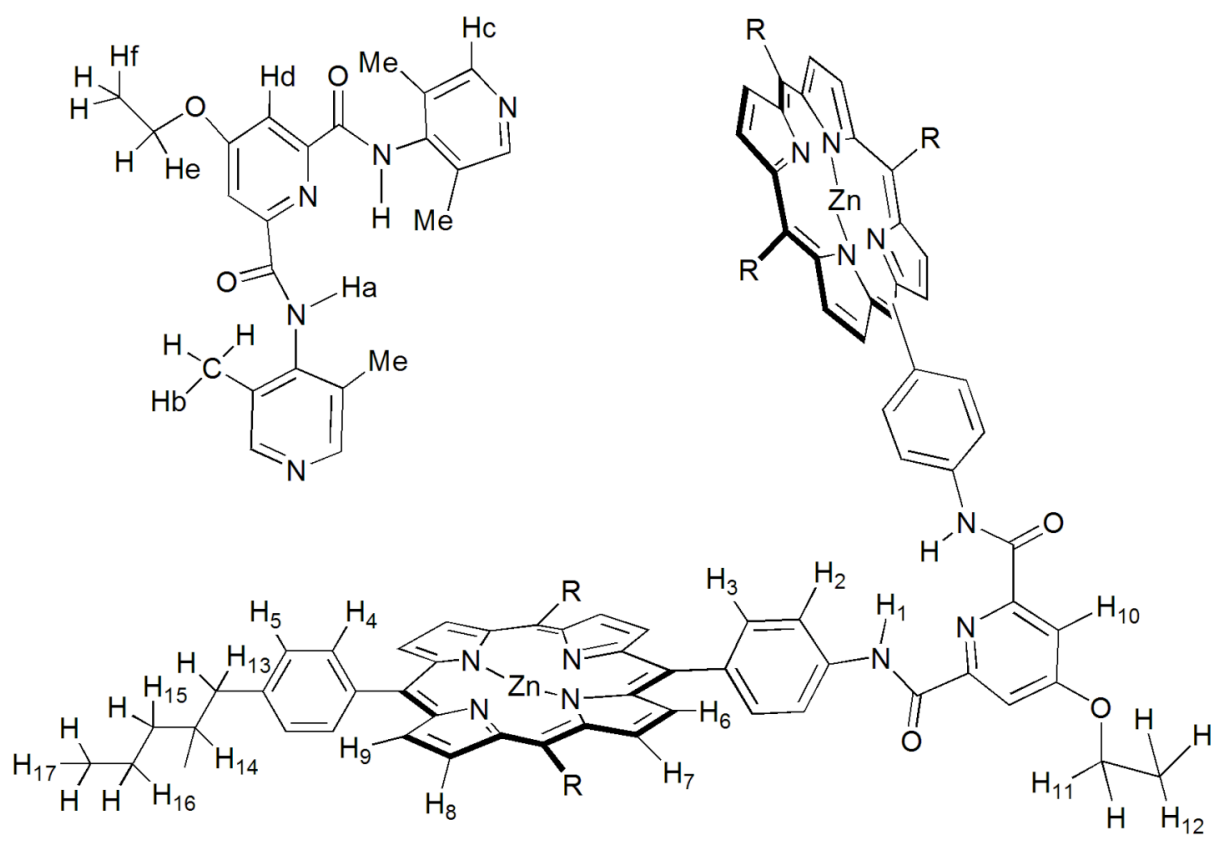

2. Figure S2. Selected regions of the ${ }^{1} \mathrm{H}$ NMR spectra recorded during a titration of zinc bisporphyrin 4a with bispyridine 3a in $\mathrm{CDCl}_{3}$ at $295 \mathrm{~K}$ (0-4.3 equiv of 3a are added) and proton assignment. Signals labeled $\mathrm{Hc}$ and $\mathrm{Hb}$ represent the proton alfa to the nitrogen and the methyl protons of the pyridine unit of $\mathbf{3 a}$ when bound as a component of $1 \mathbf{a a}$. The chemical shifts of signals $\mathrm{Hc}$ and $\mathrm{Hb}$ in free 3a are 8.27 and $2.2 \mathrm{ppm}$ respectively. When more than 1 equiv of $\mathbf{3 a}$ is added these signals broaden due to chemical exchange. 


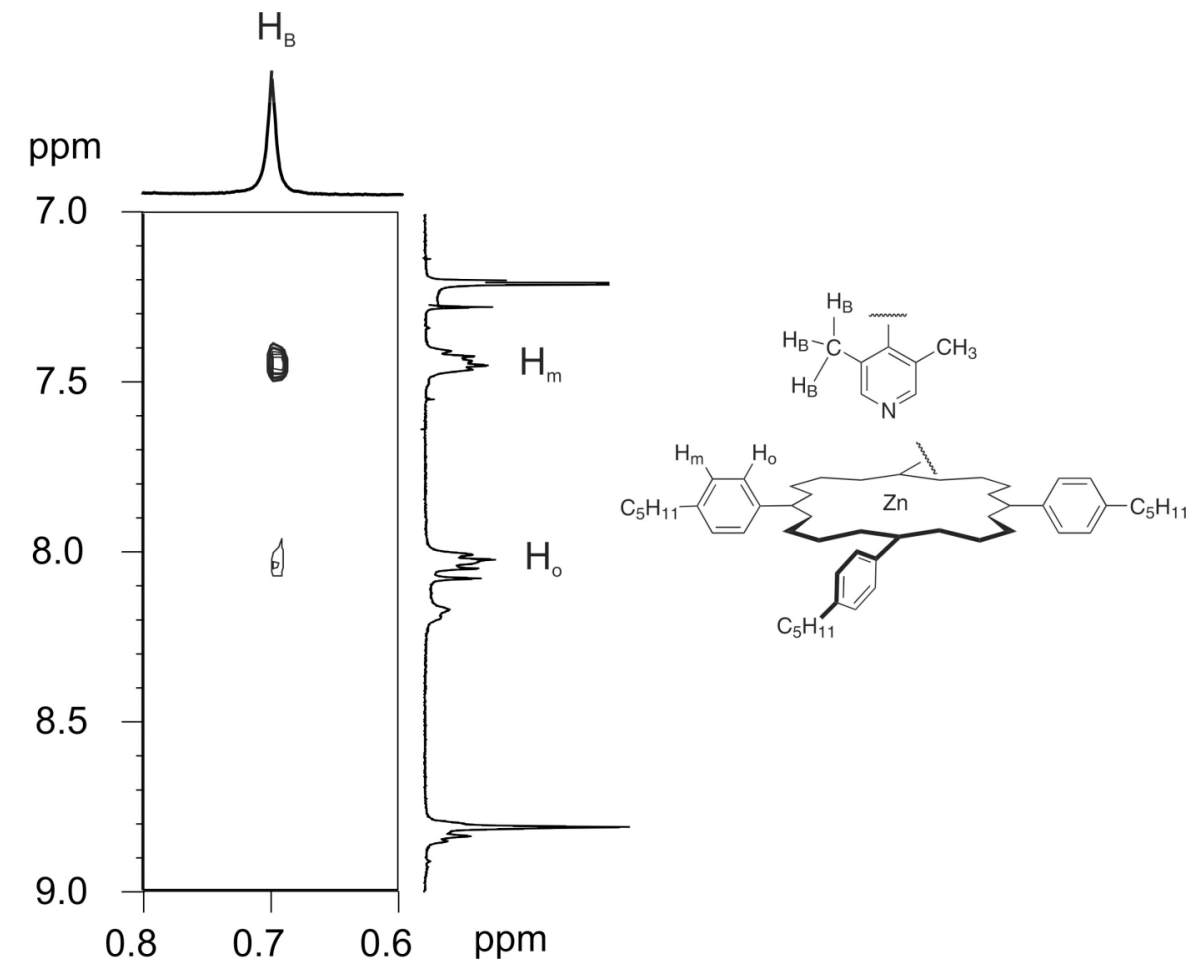

Figure S3. Expansion of a ROESY experiment performed on the self-assembled heterodimeric macrocycle 1aa. Intermolecular cross peaks between the protons of the pyridine methyl group of component 3a and the aromatic protons of the meso-phenyl susbtituens on the porphyrin ring of component $\mathbf{4 a}$ are clearly observed. This result implies spatial close proximity of these protons and is consistent with the proposed structure for 1aa. 


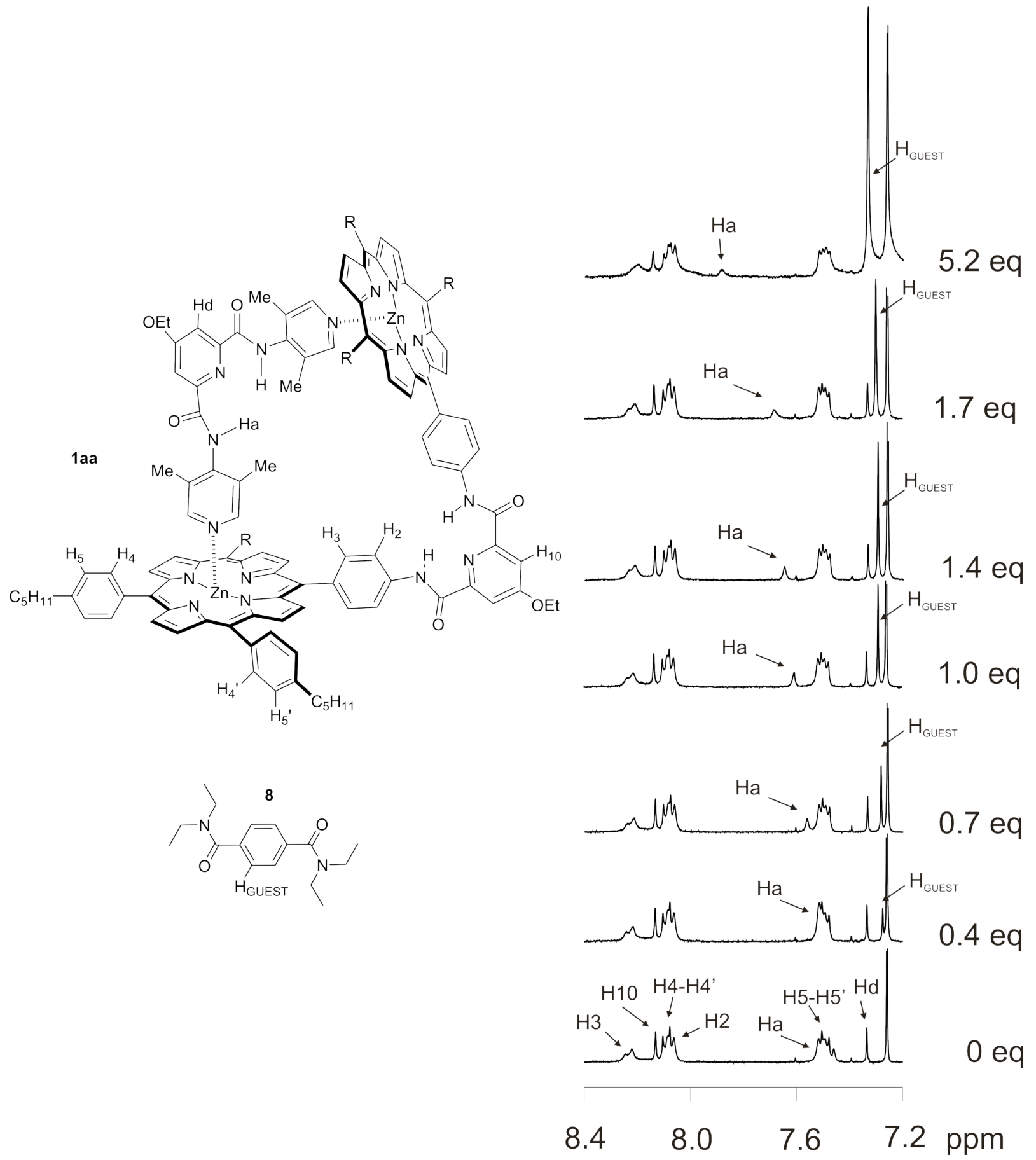

Figure S4. Aromatic region of the ${ }^{1} \mathrm{H}$ NMR spectra recorded during a titration of 1aa with diamide 8 ( $0-5.2$ equiv of $\mathbf{8}$ are added). The signal labeled as Ha corresponds to the $\mathrm{NH}$ protons of the bispyridyl (3a) unit of macrocycle 1aa. The signal labeled as $\mathrm{H}_{\mathrm{GUEST}}$ is assigned to the aromatic protons of $\mathbf{8}$. 


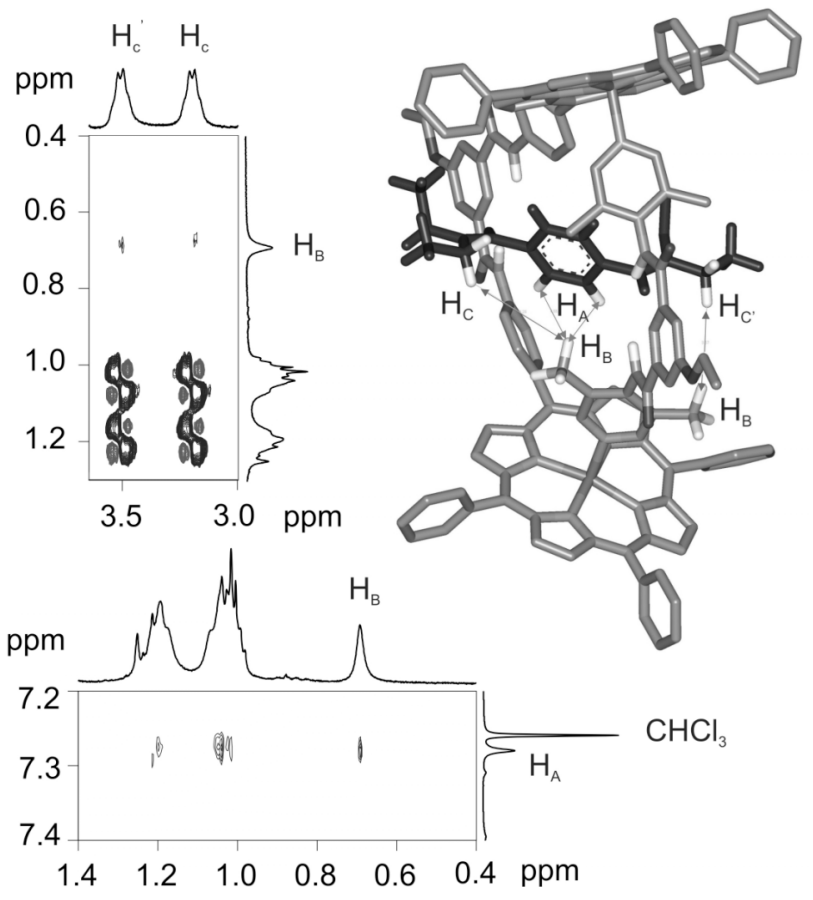

Figure S5. Selected expansions of a ROESY experiment peformed on the 1:1 complex formed by macrocycle $\mathbf{1 a a}$ and diamide $\mathbf{8}$ and the proposed geometry for the complex. Proton assignment is indicated in the structure of the complex. Intermolecular NOE crosspeaks were observed between the pyridine methyl protons $\left(\mathrm{H}_{\mathrm{B}}\right)$ of macrocycle 1aa and aromatic $\left(\mathrm{H}_{\mathrm{A}}\right)$, as well as, $\mathrm{N}$-methylene protons $\left(\mathrm{H}_{\mathrm{C}}, \mathrm{H}_{\mathrm{C}}{ }^{\prime}\right)$ of diamide 8. COSY-type artifacts (anti-phase) crosspeaks due to the scalar coupled diethyl spin system of $\mathbf{8}$ are also shown. 

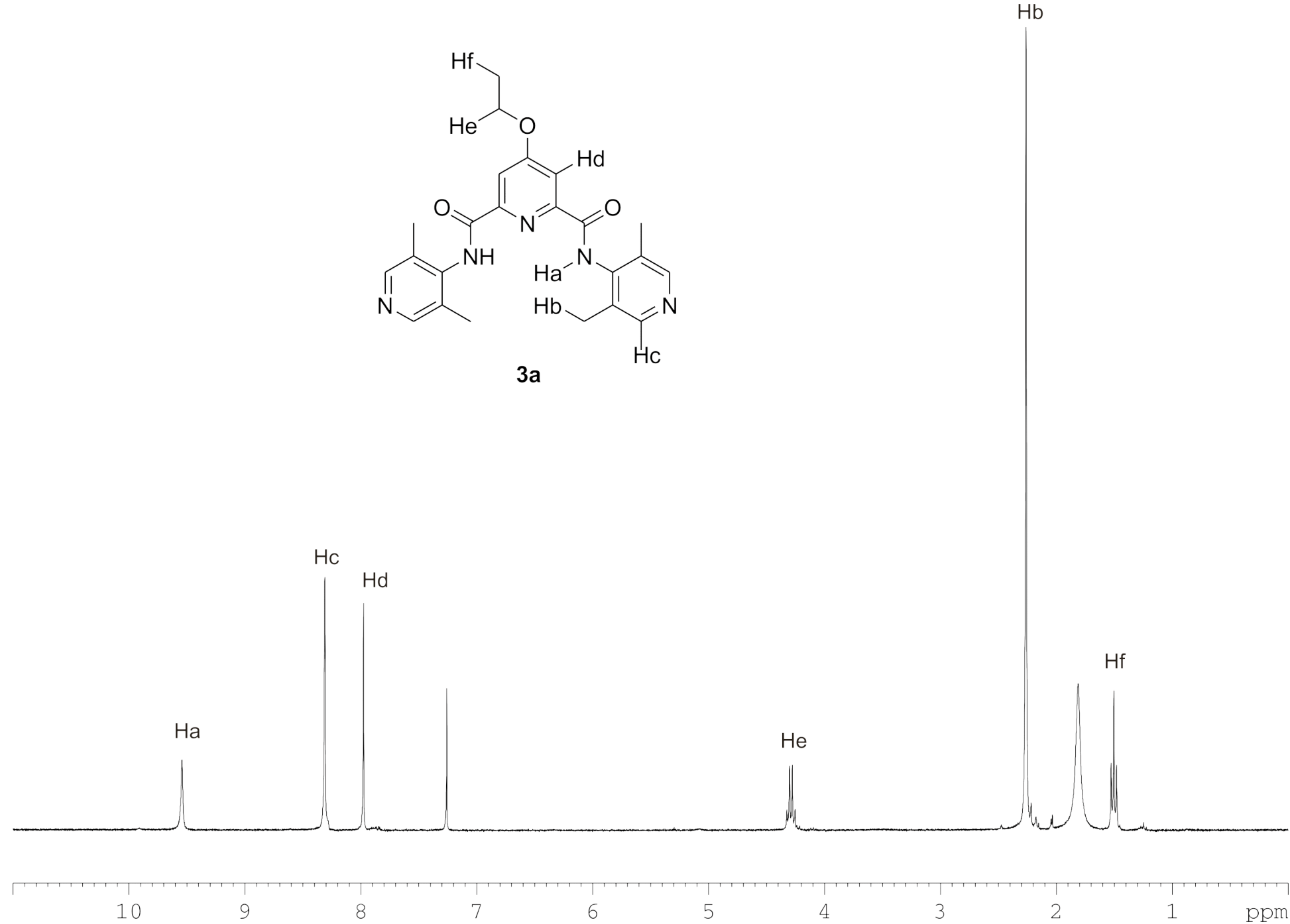

Figure S6. ${ }^{1} \mathrm{H}-\mathrm{NMR}$ spectrum of $\mathbf{3 a}$ in $\mathrm{CDCl}_{3}$ 


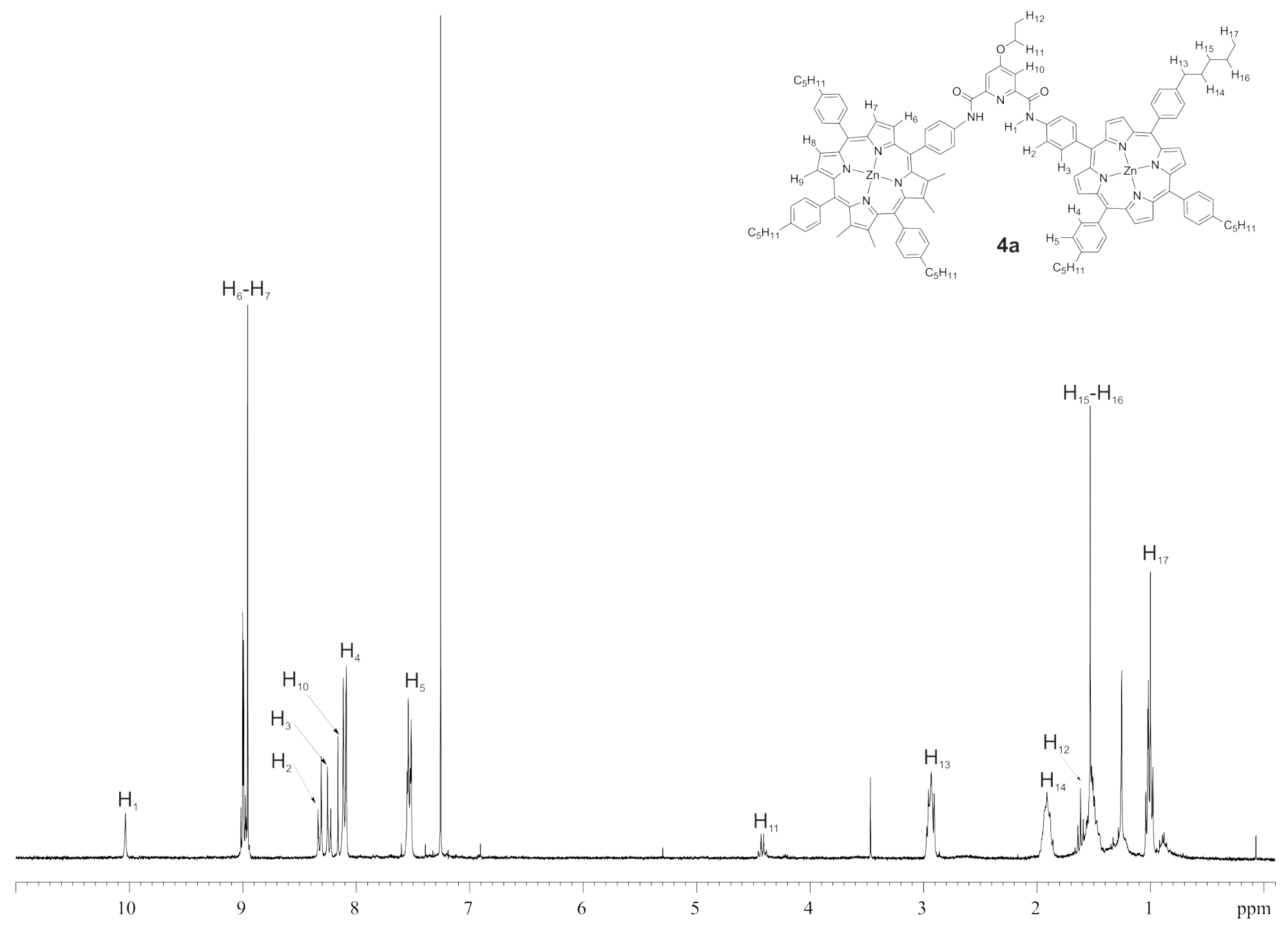

Figure S7. ${ }^{1} \mathrm{H}-\mathrm{NMR}$ spectrum of $\mathbf{4 a}$ in $\mathrm{CDCl}_{3}$ 


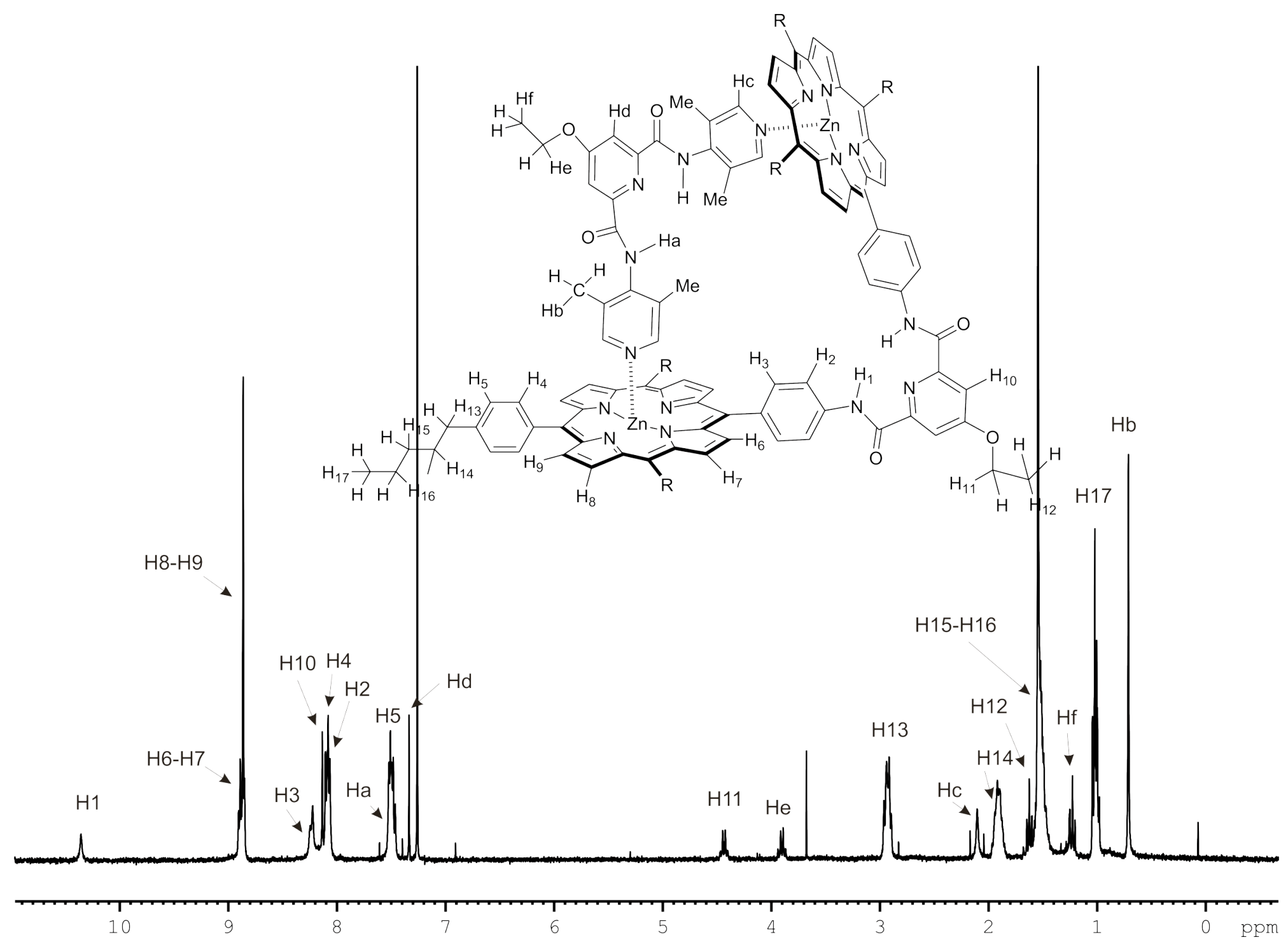

Figure S8. ${ }^{1} \mathrm{H}-\mathrm{NMR}$ spectrum of $\mathbf{1 a a}$ in $\mathrm{CDCl}_{3}$ 